\title{
ДИФФЕРЕНЦИАЛЬНЫЕ ВКЛЮЧЕНИЯ В БАНАХОВОМ ПРОСТРАНСТВЕ С СОСТАВНОЙ ПРАВОЙ ЧАСТЬЮ
}

\begin{abstract}
А. А. Толстоногов
В сепарабельном банаховом пространстве рассматривается дифференциальное включение, правая часть которого является суммой двух многозначных отображений. Первое отображение имеет своими значениями замкнутые, ограниченные, не обязательно выпуклые множества. Оно измеримо по временной переменной, является липшицевым по фазовой переменной и удовлетворяет традиционному условию роста. Второе многозначное отображение в качестве своих значений имеет замкнутые, выпуклые, не обязательно ограниченные множества. Предполагается, что это отображение имеет по фазовой переменной замкнутый график. Остальные предположения относятся к пересечению второго отображения и многозначного отображения, определенного условиями роста. Считается, что пересечение многозначных отображений имеет измеримый селектор и обладает определенными свойствами компактности. Доказана теорема существования решений таких включений. Доказательство базируется на принадлежащей автору теореме о непрерывных селекторах, проходящих через неподвижные точки многозначных отображений, зависящих от параметра, с замкнутыми, невыпуклыми, разложимыми значениями и классической теореме Ки Фана о неподвижной точке. Полученные результаты являются новыми.
\end{abstract}

Ключевые слова: разложимое множество, неподвижная точка, непрерывный селектор, слабая норма, интеграл Аумана.

A. A. Tolstonogov. Differential inclusions in a Banach space with composite right-hand side.

A differential inclusion whose right-hand side is the sum of two multivalued mappings is considered in a separable Banach space. The values of one mapping are closed, bounded, not necessarily convex sets. This mapping is measurable in the time variable, is Lipschitz in the state variable, and satisfies the traditional growth condition. The values of the second mapping are closed, convex, not necessarily bounded sets. This mapping is assumed to have a closed graph in the state variable. The remaining assumptions concern the intersection of the second mapping and the multivalued mapping defined by the growth conditions. We suppose that the intersection of the multivalued mappings has a measurable selection and possesses certain compactness properties. An existence theorem is proved for solutions of such inclusions. The proof is based on a theorem proved by the author on continuous selections passing through fixed points of multivalued mappings depending on a parameter with closed, nonconvex, decomposable values and on Ky Fan's famous fixed-point theorem. The obtained results are new.

Keywords: decomposable space, fixed point, continuous selection, weak norm, Aumann integral.

MSC: $58 \mathrm{C} 06$

DOI: $10.21538 / 0134-4889-2020-26-1-212-222$

\section{1. Введение}

Пусть $T=[0, a], a>0,-$ отрезок числовой полупрямой $R^{+}=[0,+\infty)$ с мерой Лебега и с $\sigma$-алгеброй $\Sigma$ измеримых по Лебегу множеств из $T, X$ - сепарабельное банахово пространство с нормой $\|\cdot\|$ и с нулевым элементом $\Theta, B$ - открытый единичный шар с центром в $\Theta, \bar{B}-$ его замыкание. Через $d(x, D)$ мы обозначаем расстояние от точки $x$ до множества $D \subset X$ и

$$
\|D\|=\sup \{\|x\| ; x \in D\} .
$$

Обозначим через $L^{1}(T, X)$ пространство интегрируемых по Бохнеру функций из $T$ в $X$, а через $A^{1,1}(T, X)$ - пространство всех абсолютно непрерывных функций $x: T \rightarrow X$, которые имеют производные $\dot{x}(\cdot) \in L^{1}(T, X)[1]$.

Метрику Хаусдорфа на пространстве всех непустых, замкнутых, ограниченных множеств из $X$ мы обозначаем как haus $(\cdot, \cdot)$. 
Целью работы является доказательство существования решения дифференциального включения

$$
\dot{x} \in U(t, x)+V(t, x), x(0)=x_{0},
$$

где $U, V: T \times X \rightarrow X-$ многозначные отображения.

Под решением включения (1.1) понимается тройка $(x(u, v)(\cdot), u(\cdot), v(\cdot))$,

$$
x(u, v)(\cdot) \in A^{1,1}(T, X), \quad x(u, v)(0)=x_{0}, \quad u(\cdot) \in L^{1}(T, X), \quad v(\cdot) \in L^{1}(T, X),
$$

удовлетворяющая почти всюду

$$
\begin{gathered}
\dot{x}(u, v)(t)=u(t)+v(t), \\
u(t) \in U(t, x(u, v)(t)), \\
v(t) \in V(t, x(u, v)(t)) .
\end{gathered}
$$

Сделаем следующие предположения.

$\Gamma$ и п о т е з ы $H(U)$. Многозначное отображение $U: T \times X \rightarrow X$ с непустыми, замкнутыми, не обязательно выпуклыми, значениями обладает следующими свойствами:

1) при всех $x \in X$ отображение $t \rightarrow U(t, x)$ измеримо;

2) имеют место неравенства

$$
\operatorname{haus}(U(t, x), U(t, y)) \leq k(t)\|x-y\| \text { п.в. }
$$

$x, y \in X, k(\cdot) \in L^{1}\left(T, R^{+}\right)$,

$$
\|U(t, x)\|=\sup \{\|u\| ; u \in U(t, x)\} \leq m_{1}(t)+n_{1}(t)\|x\| \text { п.в. }, \quad x \in X,
$$

$m_{1}(\cdot), n_{1}(\cdot) \in L^{1}\left(T, R^{+}\right)$.

$\Gamma$ и п о т е з ы $H(V)$. Многозначное отображение $V: T \times X \rightarrow X$ с непустыми, выпуклыми, замкнутыми значениями обладает следующими свойствами:

1) выполняется неравенство

$$
d(\Theta, V(t, x))<m_{2}(t)+n_{2}(t)\|x\| \text { п.в. },
$$

$m_{2}(\cdot), n_{2}(\cdot) \in L^{1}\left(T, R^{+}\right)$

2) при каждом $x \in X$ отображение

$$
t \rightarrow V(t, x) \cap\left(m_{2}(t)+n_{2}(t)\|x\|\right) \bar{B}
$$

имеет измеримый селектор, а отображение $x \rightarrow V(t, x)$ имеет замкнутый график для почти каждого $t \in T$;

3) для каждого ограниченного множества $D \subset X$ множество

$$
V(t, D) \cap\left(m_{2}(t)+n_{2}(t)\|D\|\right) \bar{B}
$$

относительно компактно при почти всех $t \in T$, где $V(t, D)=\{\cup V(t, x) ; x \in D\}$.

Так как в (1.7) неравенство строгое, то для почти каждого $t$ множество

$$
V(t, x) \cap\left(m_{2}(t)+n_{2}(t)\|x\|\right) \bar{B}, \quad x \in X,
$$

не пусто. Поэтому гипотеза $H(V) 2)$ носит содержательный характер.

Основной результат работы составляет теорема.

Теорема 1. Пусть выполняются гипотезы $H(U)$ и $H(V)$. Тогда дифференциальное включение (1.1) имеет решение. 
Отметим, что дифференциальное включение (1.1) с правой частью составного типа изучается впервые.

Если $V(t, x) \equiv \Theta, t \in T, x \in X$, то как следствие мы получаем теорему существования решения дифференциального включения с невыпуклозначной липшицевой правой частью. Наиболее общие из известных результатов в этом направлении получены в работе [2].

Если $U(t, x) \equiv \Theta, t \in T, x \in X$, то мы получаем теорему существования решения дифференциального включения, значениями правой части которого являются замкнутые, выпуклые множества.

Как следует из неравенства (1.7), значениями отображения $V(t, x)$ могут быть неограниченные множества.

Во всех известных результатах (см. [3] и др.), относящихся к такому включению, наряду с другими предположениями считается, что значениями отображения $V(t, x)$ являются выпуклые компактные множества, а отображение $x \rightarrow V(t, x)$ полунепрерывно сверху. Поскольку в конечномерном пространстве из неравенства (1.7) гипотеза $H(V) 3)$ следует автоматически, то даже в этом случае при $U(t, x) \equiv \Theta, t \in T, x \in X$ теорему 1 можно считать новой.

\section{2. Основные обозначения, определения и вспомогательные результаты}

Пусть $Y$ - метрическое пространство, $c Y$ - семейство всех непустых замкнутых множеств из $Y, c b Y$ - семейство всех ограниченных множеств из $c Y$ с метрикой Хаусдорфа haus $(\cdot, \cdot)$. Для топологического векторного пространства $Z$ через $\omega-Z$ мы будем обозначать пространство $Z$, наделенное слабой топологией. Если $D \subset Z$, то $\omega-D$ означает, что $D$ наделено топологией, индуцированной топологией пространства $\omega-Z$. Под $\overline{c o} D$ мы понимаем замкнутую выпуклую оболочку множества $D \subset Z$.

Пусть $W$ - топологическое пространство. Многозначное отображение $F: W \rightarrow Y$ называется полунепрерывным снизу, если для любого открытого множества $E \subset Y$ множество $F^{-1}(E)=\{w \in W ; F(w) \cap E \neq \varnothing\}$ открыто.

Многозначное отображение $F: W \rightarrow Y$ называется полунепрерывным сверху, если для любого открытого множества $E \subset Y$ множество $F^{+}(E)=\{w \in W ; F(w) \subset E\}$ открыто.

Отметим, что если $W$ - метрическое пространство, то определение полунепрерывности снизу эквивалентно следующему: для любых $w \in W, y \in F(w)$ и любой последовательности $w_{n} \in W, n \geq 1, w_{n} \rightarrow w$ существует последовательность $y_{n} \in F\left(w_{n}\right), n \geq 1$, сходящаяся к $y$.

Если $Y$ - компактное метрическое пространство, $W$ - метрическое пространство и $F$ : $W \rightarrow Y$ - многозначное отображение с замкнутыми значениями, то полунепрерывность сверху эквивалентна замкнутости графика отображения $F$.

Многозначное отображение $F: T \rightarrow c X$ называется измеримым [4], если множество $F^{-1}(E)=\{t \in T ; F(t) \cap E \neq \varnothing\}$ является измеримым для любого замкнутого множества $E \subset X$.

Множество $\mathcal{K}$ измеримых отображений из $T$ в $X$ называется разложимым, если для любых $u, v \in \mathcal{K}, E \in \Sigma$ элемент $\chi(E) u+\chi(T \backslash E) v$ принадлежит множеству $\mathcal{K}$, где $\chi(E)$ - характеристическая функция множества $E$.

На пространстве $L^{1}(T, X)$, наряду со слабой топологией, рассмотрим топологию, порожденную так называемой "слабой” нормой [5]:

$$
\left|\|f\|_{L^{1}}\right|=\max _{t \in T}\left\|\int_{0}^{t} f(s) d s\right\|, \quad f(\cdot) \in L^{1}(T, X) .
$$

Пространство $L^{1}(T, X)$ с нормой $(2.1)$ обозначается через $|\omega|-L^{1}(T, X)$.

Как обычно, $C(T, X)$ - это пространство всех непрерывных отображений из $T$ в $X$ с топологией равномерной сходимости на $T$. 
Измеримое многозначное отображение $\Gamma: T \rightarrow c b X$ назовем интегрально ограниченным, если существует функция $m(\cdot) \in L^{1}\left(T, R^{+}\right)$такая, что

$$
\|\Gamma(t)\|=\sup \{\|u\| ; u \in \Gamma(t)\} \leq m(t) \text { п.в. }
$$

Пространство всех измеримых, интегрально ограниченных отображений $\Gamma: T \rightarrow c b X$ будем обозначать через $L^{1}(T, c b X)$, а под $d c b L^{1}(T, X)$ мы понимаем совокупность всех замкнутых, ограниченных, разложимых множеств из $L^{1}(T, X)$.

Если $\Gamma(\cdot) \in L^{1}(T, c b X)$, то $S_{\Gamma}$ - совокупность всех интегрируемых селекторов отображения $t \rightarrow \Gamma(t)$, которое, как хорошо известно, является элементом пространства $d c b L^{1}(T, X)$.

Теорема 2. Пусть множество $\mathcal{K} \subset L^{1}(T, X)$ обладает следующими свойствами:

1) для почти каждого $t \in T$ множество $\{f(t) ; f(\cdot) \in \mathcal{K}\} \subset X$ относительно компактно;

2) существует функиия $m(\cdot) \in L^{1}\left(T, R^{+}\right)$такая, что $\|f(t)\| \leq m(t)$ n.в. для любого $f(\cdot) \in \mathcal{K}$.

Тогда на множестве $\mathcal{K}$ топологии пространств $\omega-L^{1}(T, X)$ u $|\omega|-L^{1}(T, X)$ cовпадают.

Д о к а з а т е л ь с т в о. Пусть $S \subset \mathcal{K}-$ счетное плотное подмножество и $\Gamma: T \rightarrow X-$ многозначное отображение, определенное по правилу

$$
\Gamma(t)=\overline{\{\cup f(t) ; f(\cdot) \in S\}}, \quad t \in T,
$$

где черта вверху означает замыкание в $X$. Тогда $\Gamma(\cdot) \in L^{1}(T, c b X)$ и для любого $f(\cdot) \in \mathcal{K}$ справедливо включение $f(t) \in \Gamma(t)$ п.в.

Рассмотрим линейный оператор $\mathcal{L}: L^{1}(T, X) \rightarrow C(T, X)$

$$
\mathcal{L}(f)(t)=\int_{0}^{t} f(\tau) d \tau, \quad t \in T
$$

который является непрерывным из $\omega-L^{1}(T, X)$ в $\omega-C(T, X)$.

Пусть $S_{\overline{\text { со Г }}}-$ множество интегрируемых селекторов многозначного отображения $t \rightarrow \overline{\mathrm{co}} \Gamma(t)$, которое является измеримым, интегрально ограниченным с выпуклыми компактными значениями. Поэтому множество $S_{\overline{\text { со }}}$ есть выпуклое компактное подмножество пространства $\omega-L^{1}(T, X)$, а множество $\mathcal{L}\left(S_{\overline{\mathrm{co}} \Gamma}\right)$ - выпуклое компактное подмножество пространства $\omega-C(T, X)$. Пусть $(\mathcal{A}) \int_{0}^{t} \overline{\mathrm{co}} \Gamma(s) d s, t \in T$, - интеграл Аумана от многозначного отображения со $\Gamma(t)$, который существует, и его значениями являются выпуклые компактные множества в пространстве $X[3]$. Из определения интеграла Аумана следует, что

$$
\mathcal{L}(f)(t) \in(\mathcal{A}) \int_{0}^{t} \overline{\mathrm{co}} \Gamma(s) d s, \quad t \in T, \quad f(\cdot) \in S_{\overline{\mathrm{co}} \Gamma} .
$$

Из включения $(2.2)$, компактности множеств $(\mathcal{A}) \int_{0}^{t} \overline{c 0} \Gamma(s) d s, t \in T$, интегральной ограниченности отображения $t \rightarrow \overline{\mathrm{co}} \Gamma(t)$, замкнутости множества $\mathcal{L}\left(S_{\overline{\mathrm{co}} \Gamma}\right)$ в пространстве $C(T, X)$ и теоремы Арцела - Асколи вытекает, что множество $\mathcal{L}\left(\overline{\mathrm{co}} S_{\overline{\mathrm{co}} \Gamma}\right)$ есть выпуклый компакт в пространстве $C(T, X)$. Тогда сужение отображения $\mathcal{L}$ на множество $S_{\overline{\text { сог }}}$ является непрерывной

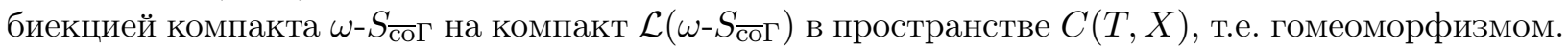
Воспользовавшись $(2.1)$ и определением нормы на пространстве $C(T, X)$, мы получим, что на множестве $S_{\overline{\text { сог }}}$ топологии пространств $\omega-L^{1}(T, X)$ и $|\omega|-L^{1}(T, X)$ совпадают. Так как $\mathcal{K} \subset S_{\overline{\text { сог }} \text {, }}$ то теорема доказана.

Функцию $x: T \rightarrow X$ назовем кусочно-постоянной и непрерывной справа, если существует разбиение $0=t_{0}<t_{1}<\ldots t_{n}=a$ отрезка $T$ такое, что на каждом из полуинтервалов $\left[t_{i-1}, t_{i}\right), 1 \leq i \leq n$, функция $x(t)$ постоянна. 
Теорема 3. Для любой непрерывной функиии $x: T \rightarrow X$ многозначные отображения

$$
\begin{aligned}
t & \rightarrow U(t, x(t)), \\
t \rightarrow V(t, x(t)) & \cap\left(m_{2}(t)+n_{2}(t)\|x(t)\|\right) \bar{B}
\end{aligned}
$$

имеют интегрируемые селекторы.

Д о к а з а т е л ь с т в о. Из гипотез $H(U) 1), 2$ ) следует, что многозначное отображение $t \rightarrow U(t, x(t))$ является измеримым с замкнутыми значениями. Поэтому существует измеримый селектор [4] этого отображения, который согласно гипотезе $H(U) 3)$ является элементом пространства $L^{1}(T, X)$.

Докажем существование интегрируемого селектора у отображения (2.3). Пусть

$$
F(t, x)=V(t, x) \cap\left(m_{2}(t)+n_{2}(t)\|x(t)\| \bar{B} .\right.
$$

Из гипотез $H(V)$ следует, что значениями отображения $F(t, x)$ являются выпуклые компактные множества и отображение $t \rightarrow F(t, x)$ имеет измеримый селектор.

Пусть $x(\cdot) \in C(T, X)$. Воспользовавшись теоремой 1.5 и следствием 1.2 работы [6], мы получаем, что существует последовательность $x_{n}(\cdot), n \geq 1$, кусочно постоянных, непрерывных справа функций, сходящаяся равномерно к $x(\cdot)$, и множество $\cup_{n=1}^{\infty}\left\{x_{n}(t) ; t \in T\right\}$ относительно компактно в $X$.

Тогда для каждой функции $x_{n}(\cdot), n \geq 1$, существует измеримая функция $f_{n}: T \rightarrow X$ такая, что

$$
f_{n}(t) \in F\left(t, x_{n}(t)\right) \text { п.в. }
$$

Поскольку множество $\cup_{n=1}^{\infty}\left\{x_{n}(t) ; t \in T\right\} \subset X$ относительно компактно, то существует компактное множество $C \subset X$ такое, что

$$
x(t) \cup\left\{\cup x_{n}(t) ; n \geq 1\right\} \subset C, \quad t \in T .
$$

Из гипотезы $H(V) 3)$ вытекает, что значениями многозначного отображения $t \rightarrow \overline{\operatorname{co}} V(t, C)$ при почти каждом $t \in T$ являются выпуклые компактные множества и

$$
\begin{gathered}
F(t, x) \subset \overline{\mathrm{co}} V(t, C), \text { п.в., } \quad x \in C, \\
f_{n}(t) \in \overline{c o} F(t, C) \subset\left(m_{2}(t)+n_{2}(t)\|C\|\right) \bar{B} \text { п.в. }
\end{gathered}
$$

Из (2.6) и гипотезы $H(V) 2)$ выводим, что сужение отображения $x \rightarrow F(t, x), t \in T$, на множество $C$ полунепрерывно сверху при почти каждом $t \in T$. Согласно (2.7) последовательность $f_{n}(\cdot), n \geq 1$, является относительно компактным подмножеством пространства $\omega-L^{1}(T, X)$. Так как пространство $L^{1}(T, X)$ сепарабельно, то любой компакт в пространстве $\omega-L^{1}(T, X)$ метризуем. Поэтому из последовательности $f_{n}(\cdot), n \geq 1$, можно выбрать подпоследовательность $f_{n_{k}}(\cdot), k \geq 1$, сходящуюся в пространстве $\omega-L^{1}(T, X)$ к некоторой функции $f(\cdot) \in L^{1}(T, X)$.

Чтобы избежать новых обозначений, не нарушая общности, будем считать, что последовательность $f_{n}(\cdot), n \geq 1$, сама сходится в пространстве $\omega-L^{1}(T, X)$ к $f(\cdot)$. Тогда из $(2.5)$, полунепрерывности сверху на $C$ отображения $x \rightarrow F(t, x)$ и леммы Мазура о слабо сходящихся последовательностях мы получим

$$
f(t) \in \cap_{n \geq 1} \overline{\mathrm{CO}}\left\{\cup_{k \geq n} F\left(t, x_{k}(t)\right)\right\} \subset F(t, x(t)) \text { п.в. }
$$

Следовательно, $f(t)$ - интегрируемый селектор отображения (2.4). Теорема доказана.

Лемма 1. Пусть $\Gamma_{i}(\cdot) \in L^{1}(T, c b X), i=1,2$. Тогда

$$
\operatorname{haus}_{L^{1}}\left(S_{\Gamma_{1}}, S_{\Gamma_{2}}\right) \leq \int_{T} \operatorname{haus}\left(\Gamma_{1}(t), \Gamma_{2}(t)\right) d t .
$$


Д о к а з а т е л ь с т в о. Лемма 1 вытекает из утверждения 4.2 в [7].

Пусть $r(t), t \in T$ - решение дифференциального уравнения

$$
\dot{r}(t)=m(t)+n(t) r(t), \quad r(0)=r_{0}, \quad m(\cdot), n(\cdot) \in L^{1}\left(T, R^{+}\right) .
$$

Лемма 2. Если функиия $x(\cdot) \in C(T, X)$ удовлетворяет неравенству

$$
\|x(t)\| \leq r_{0}+\int_{0}^{t}(m(\tau)+n(\tau)\|x(\tau)\|) d \tau, \quad t \in T,
$$

mo

$$
\|x(t)\| \leq r(t), \quad t \in T .
$$

Д о к а з а т е л ь с т в о. Лемма непосредственно вытекает из свойств дифференциальных неравенств (см., например, теорему I.1.7 в [3]).

\section{3. Многозначный оператор Немыцкого}

Рассмотрим дифференциальное уравнение

$$
\begin{gathered}
\dot{x}(t)=u(t)+v(t), \quad x(0)=x_{0}, \\
u(\cdot), v(\cdot) \in L^{1}(T, X) .
\end{gathered}
$$

Обозначим через $x(u ; v)$ решение уравнения (3.1), которое имеет вид

$$
x(u ; v)(t)=x_{0}+\int_{0}^{t} u(s) d s+\int_{0}^{t} v(s) d s .
$$

Обозначим через $\mathcal{T}(u ; v)$ оператор, который любым $u(\cdot), v(\cdot) \in L^{1}(T, X)$ ставит в соответствие единственное решение $x(u ; v)$ уравнения (3.1), т. е.

$$
x(u ; v)(t)=\mathcal{T}(u ; v)(t) .
$$

Лемма 3. Оператор $\mathcal{T}(u ; v)$ является непрерывным отображением

$$
\text { uз } L^{1}(T, X) \times|\omega|-L^{1}(T, X) \quad \text { в } C(T, X) .
$$

Д о к а з а т е л ь с т в о. Лемма с очевидностью вытекает из (3.2), (2.1) и определения нормы в пространстве $L^{1}(T, X)$.

Пусть $u(\cdot), v(\cdot) \in L^{1}(T, X)$. Рассмотрим многозначное отображение $t \rightarrow U(t, \mathcal{T}(u ; v)(t))$. Из гипотез $H(U)$ следует, что отображение $t \rightarrow U(t, \mathcal{T}(u ; v)(t))$ есть элемент пространства $L^{1}(T, c b X)$.

Поэтому множество

$$
\Phi(u ; v)=\left\{f(\cdot) \in L^{1}(T, X) ; f(t) \in U(t, \mathcal{T}(u ; v)(t)) \text { п.в. }\right\}
$$

является элементом пространства $d c b L^{1}(T, X)$. Тем самым будет определено многозначное отображение $\Phi: L^{1}(T, X) \times L^{1}(T, X) \rightarrow d c b L^{1}(T, X)$, которое называется многозначным оператором Немыцкого.

На пространстве $L^{1}(T, X)$ рассмотрим числовую функцию

$$
P(x)=\int_{T} \rho(t, x(t)) d t
$$


где

$$
\rho(t, x(t))=\left(\exp \left(-2 \int_{0}^{t} k(\tau) d \tau\right)\right)\|x(t)\|, \quad t \in T .
$$

Здесь функция $k(\cdot)$ взята из неравенства (1.5).

Очевидно, что функция $P(x)$ определяет норму, эквивалентную норме $\|x\|_{L^{1}}$ пространства $L^{1}(T, X)$.

Расстояние по Хаусдорфу между множествами из пространства $c b L^{1}(T, X)$, наделенного нормой $P(x)$, будем обозначать через $\operatorname{haus}_{P}(\cdot, \cdot)$.

Теорема 4. Оператор Немыцкого $\Phi: L^{1}(T, X) \times L^{1}(T, X) \rightarrow c b L^{1}(T, X)$ обладает следующими свойствами:

$$
\operatorname{haus}_{L^{1}}\left(\Phi\left(u_{1} ; v_{1}\right), \Phi\left(u_{2} ; v_{2}\right)\right) \leq L\left(\left\|u_{1}-u_{2}\right\|_{L^{1}}+\left|\left\|v_{1}-v_{2}\right\|_{L^{1}}\right|\right),
$$

где

$$
\begin{gathered}
L=\int_{T} k(t) d t \\
\operatorname{haus}_{P}\left(\Phi\left(u_{1} ; v\right), \Phi\left(u_{2} ; v\right)\right) \leq \frac{1}{2} P\left(u_{1}-u_{2}\right),
\end{gathered}
$$

$v \in L^{1}(T, X), u_{i} \in L^{1}(T, X), i=1,2$.

Д о к а з а т е л ь с т в о. Из (1.5), (2.9), (3.2), (3.3) мы получаем

$$
\begin{gathered}
\operatorname{haus}_{L^{1}}\left(\Phi\left(u_{1} ; v_{1}\right), \Phi\left(u_{2} ; v_{2}\right)\right) \leq \int_{T} k(\tau)\left\|\mathcal{T}\left(u_{1} ; v_{1}\right)-\mathcal{T}\left(u_{2} ; v_{2}\right)(\tau)\right\| d \tau \\
\leq \int_{T} k(\tau)\left(\left\|u_{1}-u_{2}\right\|_{L^{1}}+\left|\left\|v_{1}-v_{2}\right\|_{L^{1}}\right|\right) d \tau
\end{gathered}
$$

Теперь неравенство (3.7) вытекает из (3.10), (3.8) и (2.1).

Докажем неравенство (3.9). Из (1.5), (3.1), (3.3) выводим

$$
\operatorname{haus}\left(U\left(t, \mathcal{T}\left(u_{1} ; v\right)(t)\right), U\left(t, \mathcal{T}\left(u_{2} ; v\right)(t)\right)\right) \leq k(t) \int_{0}^{t}\left\|u_{1}(s)-u_{2}(s)\right\| d s, \quad t \in T .
$$

Воспользовавшись неравенством (3.11) и (3.4)-(3.6), имеем

$$
\operatorname{haus}_{P}\left(\Phi\left(u_{1} ; v\right), \Phi\left(u_{2} ; v\right)\right) \leq \int_{T}\left(\exp \left(-2 \int_{0}^{t} k(\tau) d \tau\right)\right) k(t)\left(\int_{0}^{t}\left\|u_{1}(s)-u_{2}(s)\right\| d s\right) d t
$$

Проинтегрировав правую часть (3.12) по частям, мы придем к неравенству

$$
\operatorname{haus}_{P}\left(\Phi\left(u_{1} ; v\right), \Phi\left(u_{2} ; v\right)\right) \leq \frac{1}{2}\left(\int_{T}\left(\exp \left(-2 \int_{0}^{t} k(\tau) d \tau\right)\right)\left\|u_{1}(t)-u_{2}(t)\right\| d t\right) .
$$

Теперь неравенство (3.9) вытекает из (3.13), (3.5), (3.6). Теорема доказана.

Для фиксированного $v(\cdot) \in L^{1}(T, X)$ обозначим через $(\operatorname{Fix} \Phi)(v)$ множество неподвижных точек оператора $\Phi(u ; v)$. 
Теорема 5. Пусть выполняются гипотезы $H(U)$. Тогда:

а) для любого $v \in L^{1}(T, X)$ множество $(\operatorname{Fix} \Phi)(v)$ не пусто;

б) существует непрерывная функиия $u:|\omega|-L^{1}(T, X) \rightarrow L^{1}(T, X)$ такая, что

$$
u(v) \in(\operatorname{Fix} \phi)(v), \quad v \in L^{1}(T, X),
$$

m.e.

$$
u(v) \in \Phi(u(v) ; v), \quad v \in L^{1}(T, X) .
$$

Д о к а з а т е л ь с т в о. Из неравенства (3.7) вытекает, что при фиксированном $u \in$ $L^{1}(T, X)$ отображение $v \rightarrow \Phi(u ; v)$ является полунепрерывным снизу из $|\omega|-L^{1}(T, X)$ в $L^{1}(T, X)$ с замкнутыми, ограниченными, разложимыми значениями. Так как пространство $|\omega|-L^{1}(T, X)$ есть сепарабельное метрическое пространством, то теорема 5 следует из теоремы 3.1 [8] и неравенства (3.9), если $v$ рассматривать как параметр.

\section{4. Априорные оценки}

Пусть

$$
m(t)=m_{1}(t)+m_{2}(t), \quad n(t)=n_{1}(t)+n_{2}(t),
$$

где $m_{i}(\cdot), n_{i}(\cdot), i=1,2$, - функции из неравенств (1.6), (1.7) и $r(t), r(0)=\left\|x_{0}\right\|$ - решение уравнения (2.10).

Обозначим

$$
\begin{aligned}
& S_{U}=\left\{u \in L^{1}(T, X) ;\|u(t)\| \leq m_{1}(t)+n_{1}(t) r(t) \text { п.в. }\right\}, \\
& S_{V}=\left\{v \in L^{1}(T, X) ;\|v(t)\| \leq m_{2}(t)+n_{2}(t) r(t) \text { п.в. }\right\} .
\end{aligned}
$$

Пусть $x(u ; v)(t), x(u ; v)(0)=x_{0}-$ решение уравнения (3.1) и

$$
\mathcal{T}\left(S_{U}, S_{V}\right)(t)=\left\{x(u ; v)(t) ; u \in S_{U}, v \in S_{V}\right\}, \quad t \in T .
$$

Воспользовавшись (4.1)-(4.4), (3.2), мы получим

$$
\left\|\mathcal{T}\left(S_{U}, S_{V}\right)(t)\right\| \leq r(t), \quad t \in T .
$$

Согласно гипотезе $H(V) 3)$ множество $V(t, r(a) \bar{B}) \cap\left(m_{2}(t)+n_{2}(t)\|r(a)\|\right) \bar{B}, t \in T$, относительно компактно при почти каждом $t \in T$. Так как решение $r(t), r(0)=\left\|x_{0}\right\|$ уравнения $(2.10)$ является неубывающей функцией, то в соответствии с (4.5) имеем $\mathcal{T}\left(S_{U}, S_{V}\right)(t) \subset r(a) \bar{B}, t \in T$.

Поэтому множество $V\left(t, \mathcal{T}\left(S_{U}, S_{V}\right)(t)\right) \cap\left(m_{2}(t)+n_{2}(t)\left\|\mathcal{T}\left(S_{U}, S_{V}\right)(t)\right\|\right) \bar{B}$ будет относительно компактным при почти всех $t \in T$.

Положим

$$
W(t)=V\left(t, \mathcal{T}\left(S_{U}, S_{V}\right)(t)\right) \cap\left(m_{2}(t)+n_{2}(t)\left\|\mathcal{T}\left(S_{U}, S_{V}\right)(t)\right\|\right) \bar{B}, \quad t \in T .
$$

Тогда многозначное отображение $t \rightarrow \overline{c o} W(t)$ при почти каждом $t \in T$ имеет своими значениями выпуклые компактные множества. С учетом (4.5), (4.6)

$$
\|\overline{\mathrm{co}} W(t)\| \leq m_{2}(t)+n_{2}(t) r(t)
$$

Пусть

$$
S_{\overline{\mathrm{co}} W}=\left\{v \in L^{1}(T, X) ; v(t) \in \overline{\mathrm{co}} W(t) \text { п.в. }\right\} .
$$

Из леммы 2 вытекает, что многозначное отображение

$$
t \rightarrow V\left(t, \mathcal{T}\left(S_{U}, S_{V}\right)(t)\right) \cap\left(m_{2}(t)+n_{2}(t)\left\|\mathcal{T}\left(S_{U}, S_{V}\right)(t)\right\|\right) \bar{B}, \quad u \in S_{U}, \quad v \in S_{V}
$$

имеет интегрируемые селекторы. 
Тогда множество $S_{\overline{\mathrm{co}} W(t)}$ обладает следующими свойствами:

а) $S_{\overline{\text { со } W}}$ есть непустое выпуклое компактное подмножеством пространства $\omega-L^{1}(T, X)$;

б) для любого $v \in S_{\overline{\mathrm{co}} W}$ имеет место неравенство $\|v(t)\| \leq m_{2}(t)+n_{2}(t) r(t)$ п.в.;

в) множество $S_{\overline{\text { со } W}}(t)=\left\{v(t) ; v \in S_{\overline{\text { со } W}}\right\} \subset X$ при почти всех $t \in T-$ компакт.

Свойства множества $S_{\overline{\mathrm{co}} W}$ вытекают из $(4.6),(4.7)$ и компактности значений отображения $t \rightarrow \overline{\mathrm{co}} W(t)$.

Согласно теореме 5 и (3.14) существует непрерывная функция $u:|\omega|-L^{1}(T, X) \rightarrow L^{1}(T, X)$ такая, что $u(v) \in \Phi(u(v) ; v), v \in L^{1}(T, X)$. Воспользовавшись (3.4), мы получим, что

$$
u(v)(t) \in U(t, \mathcal{T}(u(v) ; v)(t)) \text { п.в., } \quad v \in L^{1}(T, X) .
$$

Лемма 4. Имеют место включения

$$
S_{\overline{\mathrm{co} W}} \subset S_{V}, u\left(S_{\overline{\mathrm{co}} W}\right) \subset S_{U}
$$

Д о к а з а т е л ь с т в о. Первое включение вытекает из (4.7), (4.3). Пусть $\hat{v} \in S_{\overline{\mathrm{co}} W}$. Тогда из (1.6), (3.2), (4.8), (4.9) имеем

$$
\begin{gathered}
\|x(u(\hat{v}) ; \hat{v})(t)\| \leq\left\|x_{0}\right\|+\int_{0}^{t}\left(m_{1}(\tau)+n_{1}(\tau)\|x(u(\hat{v}) ; \hat{v})(\tau)\|\right) d \tau \\
+\int_{0}^{t}\left(m_{2}(\tau)+n_{2}(\tau) r(t)\right) d \tau, \quad t \in T .
\end{gathered}
$$

Рассмотрим дифференциальное уравнение

$$
\dot{r}_{1}(t)=\left(m_{1}(t)+m_{2}(t)+n_{2}(t) r(t)\right)+n_{1}(t) r_{1}(t), r(0)=r_{0} .
$$

Из леммы 2 и (4.11), (4.12) вытекает, что

$$
\|x(u(\hat{v}) ; \hat{v})(t)\| \leq r_{1}(t), \quad \hat{v} \in S_{\overline{\mathrm{co}} W} .
$$

Воспользовавшись (4.1), мы получаем, что решение уравнения (4.12) совпадает с решением $r(t)$ уравнения (2.10). Поэтому,

$$
\|x(u(\hat{v}), \hat{v})(t)\| \leq r(t), \quad \hat{v} \in S_{\overline{\mathrm{co}} W} .
$$

Тогда из $(4.9),(4.13)$ и (1.6) следует

$$
\|u(\hat{v})(t)\| \leq m_{1}(t)++n_{1}(t) r(t), \quad \hat{v} \in S_{\overline{\mathrm{co}} W} .
$$

Таким образом, согласно (4.2) имеет место второе включение в (4.10). Лемма доказана.

\section{5. Доказательство теоремы 1}

Рассмотрим оператор $\mathcal{T}(u(v) ; v)$ из $S_{\overline{\text { со } W}}$ в $C(T, X)$. Из свойств а)-в) множества $S_{\overline{\mathrm{co}} W}$, указанных в предыдущем разделе, и теоремы 2 вытекает, что на множестве $S_{\overline{\mathrm{co}} W}$ топологии пространств $|\omega|-L^{1}(T, X)$ и $\omega-L^{1}(T, X)$ совпадают. Поэтому отображение $u(v)$ будет непрерывным из $\omega-S_{\overline{\mathrm{co}} W}$ в $L^{1}(T, X)$. Воспользовавшись леммой 3 , мы получим, что оператор $\mathcal{T}(u(v) ; v)$ является непрерывным отображением из $\omega$ - $S_{\overline{\mathrm{co}} W}$ в $C(T, X)$.

Пусть

$$
F(t, x)=V(t, x) \cap\left(m_{2}(t)+n_{2}(t)\|x\|\right) \bar{B}
$$


и

$$
S_{F}(\mathcal{T}(u(v) ; v))=\left\{f \in L^{1}(T, X) ; f(t) \in F(t, \mathcal{T}(u(v) ; v)(t)) \text { п.в. }\right\}, \quad v \in S_{\overline{\mathrm{co}} W} .
$$

Из теоремы 3 и гипотез $H(V)$ следует, что $S_{F}(\mathcal{T}(u(v) ; v))$ является непустым, выпуклым, компактным подмножеством пространства $\omega-L^{1}(T, X)$. Согласно $(4.10),(4.6),(5.1)$ и $(5.2)$ имеет место включение

$$
S_{F}(\mathcal{T}(u(v) ; v)) \subset S_{\overline{\mathrm{co}} W}, \quad v \in S_{\overline{\mathrm{co}} W} .
$$

Тем самым будет определено многозначное отображение $v \rightarrow S_{F}(\mathcal{T}(u(v) ; v))$ с непустыми, выпуклыми, слабо компактными значениями из выпуклого, компактного, метризуемого множества $\omega-S_{\overline{\mathrm{co}} W}$ в $\omega-S_{\overline{\mathrm{co}} W}$.

Для удобства обозначим

$$
S_{F}(v)=S_{F}(\mathcal{T}(u(v) ; v)) .
$$

Пусть последовательность $v_{n} \in \omega-S_{\overline{\text { со }}}, n \geq 1$, слабо сходится к $v \in \omega-S_{\overline{\text { со }}}$. Тогда последовательность $\mathcal{T}\left(u\left(v_{n}\right) ; v_{n}\right), n \geq 1$, сходится к $\mathcal{T}(u(v) ; v)$ в пространстве $C(T, X)$. Воспользовавшись включением (5.3), по аналогии с доказательством теоремы 3 (см. (2.8)) мы получим

$$
\cap_{n=1}^{\infty} \overline{\mathrm{CO}}\left\{\cup_{k \geq n}^{\infty} F\left(t, \mathcal{T}\left(u\left(v_{k}\right) ; v_{k}\right)(t)\right)\right\} \subset F(t, \mathcal{T}(u(v) ; v)(t)) \text { п.в. }
$$

Если последовательность $f_{n} \in S_{F}\left(v_{n}\right), n \geq 1$, слабо сходится к $f$, то согласно (5.5) $f \in$ $S_{F}(v)$. Следовательно, отображение $v \rightarrow S_{F}(v), v \in \omega-S_{\overline{\mathrm{co}} W}$ имеет слабо замкнутый график. Из компактности, метризуемости множества $\omega-S_{\overline{\text { со }} W}$ и включения (5.3) вытекает, что отображение $v \rightarrow S_{F}(v), v \in \omega-S_{\overline{\text { со }}}$ является полунепрерывным сверху из $\omega-S_{\overline{\text { со }}}$ в $\omega-S_{\overline{\text { со } W}}$ с выпуклыми компактными значениями. Согласно теореме 1 Ки Фана [9] существует неподвижная точка $v_{*}$ отображения $v \rightarrow S_{F}(v)$, т. е.

$$
v_{*} \in S_{F}\left(v_{*}\right) .
$$

Положим $u_{*}=u\left(v_{*}\right), x\left(u_{*} ; v_{*}\right)=\mathcal{T}\left(u\left(v_{*}\right) ; v_{*}\right)$. Тогда из (5.6), (5.4), (5.2), (5.1), (4.9), (3.1) мы получим

$$
\begin{gathered}
\dot{x}\left(u_{*} ; v_{*}\right)(t)=u_{*}(t)+v_{*}(t), \\
u_{*}(t) \in U\left(t, x\left(u_{*} ; v_{*}\right)(t)\right) \text { п.в., } \\
v_{*}(t) \in V\left(t, x\left(u_{*} ; v_{*}\right)(t)\right) \text { п.в. }
\end{gathered}
$$

Таким образом, в соответствии с $(1.2),(1.3),(1.4)$ тройка $\left(x\left(u_{*}, v_{*}\right)(\cdot), u_{*}(\cdot), v_{*}(\cdot)\right)$ является решением включения (1.1). Теорема доказана.

\section{СПИСОК ЛИТЕРАТУРЫ}

1. Barbu V. Nonlinear semigroups and differential equations in Banach spaces. Netherlands: Springer, 1976. 352 p. ISBN 978-90-286-0205-2 .

2. Толстоногов А.А. Существование и релаксация решений дифференциальных включений с неограниченной правой частью в банаховом пространстве // Сиб. мат. журн. 2017. Т. 58, № 4(344). C. 937-953. doi: 10.17377/smzh.2017.58.419.

3. Tolstonogov A. Differential inclusions in Banach space. Dordrecht; Boston; London: Kluwer Acad. Publ., 2000. 302 p. ISBN 978-94-015-9490-5.

4. Himmelberg C.J. Measurable relations // Fund. Math. 1975. Vol. 87, no. 1. P. 53-72. doi: $10.4064 /$ fm-87-1-53-72 .

5. Alexiewicz A. Linear functionals on Denjoy-integrable functions // Colloquium Math. 1948. Vol. 1, no. 4. P. 289-293. doi: 10.4064/cm-1-4-289-293.

6. Толстоногов А.А. О некоторых свойствах пространства правильных функций // Мат. заметки. T. 35, № 6. 1984. C. 803-812.

7. Tolstonogov A.A., Tolstonogov D.A. $L_{p}$-continuous extreme selectors of multifunctions with decomposable values. Existence theorems // Set-valued Anal. 1996. Vol. 4, no. 2. P. 173-203. doi: $10.1007 / \mathrm{BF} 00425964$. 
8. Толстоногов A.A. Lp-непрерывные селекторы неподвижных точек многозначных отображений с разложимыми значениями. I. Теоремы существования // Сиб. мат. журн. 1999. Т. 40 , № 3. C. 695-709.

9. Fan Ky. Fixed point and minimax theorems in locally convex topological linear spaces // Proc. Nat. Acad Sci. USA. 1952. Vol. 38, no. 3. P. 121-126. doi: 10.1073/pnas.38.2.121.

Поступила 11.11.2019

После доработки 29.01.2020

Принята к публикации 3.02.2020

Толстоногов Александр Александрович

чл.-корр. РАН, д-р физ.-мат. наук, профессор

главный науч. сотрудник

Федеральное государственное бюджетное учреждение науки

Институт динамики систем и теории управления имени В. М. Матросова

Сибирского отделения Российской академии наук (ИДСТУ СО РАН),

г. Иркутск

e-mail: aatol@icc.ru

\section{REFERENCES}

1. Barbu V. Nonlinear semigroups and differential equations in Banach spaces. Netherlands: Springer, 1976, 352 p. ISBN: 978-90-286-0205-2 .

2. Tolstonogov A.A. Existence and relaxation of solutions to differential inclusions with unbounded righthand side in a Banach space. Siberian Math. J., 2017, vol. 58, no. 4, pp. 727-742. doi: 10.1134/S003744661704019X.

3. Tolstonogov A. Differential inclusions in Banach space. Dordrecht; Boston; London: Kluwer Acad. Publ., 2000, 302 p. ISBN: 978-94-015-9490-5.

4. Himmelberg C.J. Measurable relations. Fund. Math., 1975, vol. 87, no. 1, pp. 53-72. doi: $10.4064 / \mathrm{fm}-87-1-53-72$.

5. Alexiewicz A. Linear functionals on Denjoy-integrable functions. Colloquium Math., 1948, vol. 1, no. 4, pp. 289-293. doi: 10.4064/cm-1-4-289-293 .

6. Tolstonogov A.A. Properties of the space of proper functions. Math. Notes, 1984, vol. 35, no. 6, pp. 422-427. doi: 10.1007/BF01139944.

7. Tolstonogov A.A., Tolstonogov D.A. $L_{p}$-continuous extreme selectors of multifunctions with decomposable values. Existence theorems. Set-valued Anal., 1996, vol. 4, no. 2, pp. 173-203. doi: $10.1007 / \mathrm{BF} 00425964$.

8. Tolstonogov A.A. $L_{p}$-continuous selections of fixed points of multifunctions with decomposable values. I: Existence theorems. Sib. Math. J., 1999, vol. 40, no. 3, pp. 595-607. doi: 10.1007/BF02679768.

9. Fan Ky. Fixed point and minimax theorems in locally convex topological linear spaces. Proc. Nat. Acad Sci. USA, 1952, vol. 38, no. 2, pp. 121-126. doi: 10.1073/pnas.38.2.121.

Received November 11, 2019

Revised January 29, 2020

Accepted February 3, 2020

Aleksandr Aleksandrovich Tolstonogov, Dr. Phys.-Math. Sci., RAS Corresponding Member, Prof., Matrosov Institute for System Dynamics and Control Theory of Siberian Branch of Russian Academy of Sciences, Irkutsk, 664033 Russia, e-mail: aatol@icc.ru .

Cite this article as: A. A. Tolstonogov. Differential inclusions in a Banach space with composite righthand side, Trudy Instituta Matematiki i Mekhaniki URO RAN, 2020, vol. 26, no. 1, pp. 212-222 . 